\title{
Integrated Fresnel Mirrors for Scalable Trapped Ion Quantum Computing
}

\author{
Erik W. Streed ${ }^{1,2}$, Moji Ghadimi ${ }^{1}$, Valdis Blums ${ }^{1}$, Benjamin G. Norton ${ }^{1}$, Paul Connor ${ }^{1}$, Jason M. Amini ${ }^{3}$, \\ Curtis Volin ${ }^{3}$, Mirko Lobino ${ }^{1}$, David Kielpinski ${ }^{1}$ \\ ${ }^{I}$ Centre for Quantum Dynamics, Griffith University Brisbane QLD 4111 Australia \\ ${ }^{2}$ Institute for Glycomics, Griffith University Gold Coast QLD 4222 Australia \\ ${ }^{3}$ Georgia Tech Research Institute, Atlanta GA 30318 USA \\ *e.streed@griffith.edu.au
}

\begin{abstract}
Photonic interconnects are a bottleneck to achieving large-scale trapped ion quantum computing. We fabricated a surface trap with diffractive mirrors and achieved sub-wavelength imaging of a trapped ion and $2.0 \pm 0.5 \%$ coupling into a single-mode fiber.

OCIS codes: (270.5585) Quantum Information Processing; (270.5565) Quantum Communications; (050.1970) Diffractive Optics
\end{abstract}

\section{Introduction}

Trapped atomic ions are a leading system for implementing quantum information processing, demonstrating of all required gates operations for universal quantum computing and a roadmap to realizing large-scale implementations [1]. Micro-fabrication based ion traps (chip traps) with multiple zones that can trap and manipulate multiple ions have been realised [2], however there remains a bottleneck in efficiently coupling ion light into single mode fibers with a scalable solution. Monolithic fabrication of diffractive optics into the surface of a chip trap solves both the scalability problem and the problem of fine alignment between the trap and the optics.

\section{Experimental results}

Our chip trap (Fig. 1) is a modified version of the Georgia Tech Research Institute microwave chip trap [3]. Reflective diffractive optics were designed to collimate light from a focus at the RF node $58.6 \mu \mathrm{m}$ above the surface of the trap and patterned on the center electrode by e-beam lithograph. The optics are designed for the $369.5 \mathrm{~nm} \mathrm{Yb}^{+}$ cycling transition. Each optic was $80 \mu \mathrm{m}$ x $127 \mu \mathrm{m}$, the former dimension that of the center electrode rail width and the latter matching the standard pitch of a V-groove fiber array. The optics have an NA of 0.55 (perpendicular to rails) and 0.73 (along the rail), capturing $13.2 \%$ of the solid angle and close to the estimated optimal NA for entanglement generation per unit surface area [4]. The grating has a hybrid blaze, multi-level in the center for high efficiency and two level near the edges. The large numerical aperture results in an imaging system with a small depth of focus. To compensate for possible fabrication variation with the introduction of the Fresnel optic, the focal length for different collimator sites were stepped $\pm 2 \mu \mathrm{m}$ from the calculated focus in $1 \mu \mathrm{m}$ steps.

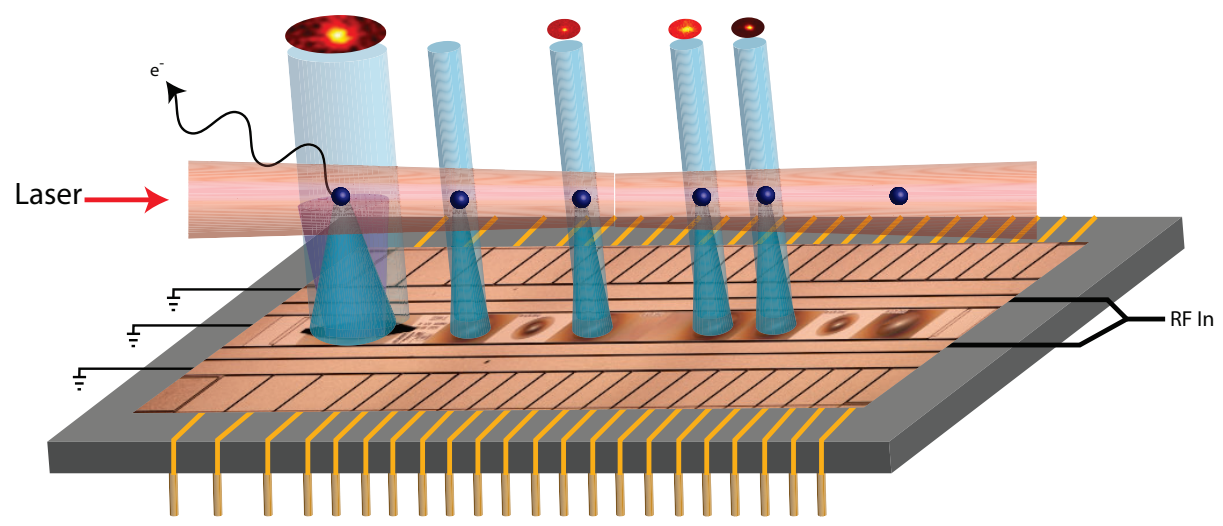

Figure 1. Georgia Tech microwave chip trap [] modified with integrated diffractive mirrors.

To evaluate the optics ${ }^{174} \mathrm{Yb}^{+}$was loaded by isotope selective photo-ionization from a thermal oven (Fig 1 left) and then shuttled to imaging sites. Near diffraction limited sub-wavelength ion images (Fig. 2) were obtained from 
the $+1 \mu \mathrm{m}$ site, with observed spot sized FWHM of $338 \mathrm{~nm}$ x $268 \mathrm{~nm}$ vs. a diffraction limit of $336 \mathrm{~nm} \times 257 \mathrm{~nm}$. To measure the collection efficiency a photon gun protocol was implemented based on an optical pumping protocol. The magnetic field direction was oriented coplanar to the chip along the direction the cooling laser propagation and at $45^{\circ}$ from the axis of the RF node. A polarizer was introduced into the imaging system to selectively detect only $\pi$ photons. By alternatingly exciting on the $\sigma+$ and $\sigma$-transitions, optical pumping ensured a sequence of $\sigma$ photons was always terminated by a $\pi$ photon as the ion was pumped into a dark state. The total photon collection efficiency was measured to be $5.2 \pm 1.2 \%$. Coupling into a single mode fibre of up to $2.0 \pm 0.5 \%$ was observed, limited by mismatch in the coupling optics. Image mode quality indicates coupling up to $\sim 4 \%$ may be possible.

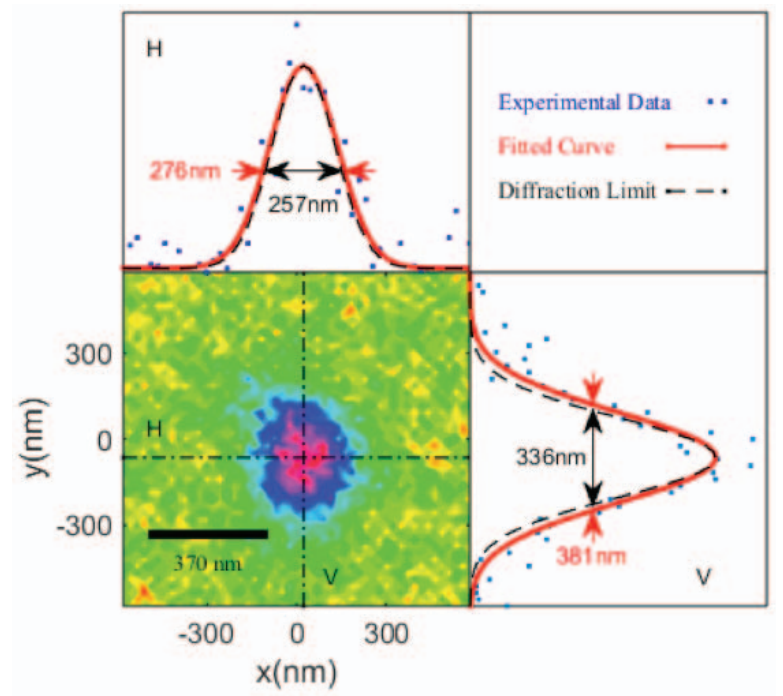

Figure 2. Sub-wavelength image of a trapped ${ }^{174} \mathrm{Yb}^{+}$ion with near diffraction-limited spot size.

\section{Conclusion}

We have demonstrated the first high-resolution imaging optics monolithically integrated on a micro-fabricated ion surface trap. Ion light was coupled into a single mode fiber. The configuration of optics used is suitable for scaling to a linear array of sites being coupled into a standard fiber array coupler.

\section{References}

[1] Monroe, C. et al. Large-scale modular quantum- computer interconnects. Phys Rev A 89, 022317 (2014)

[2] J. M. Amini et al "Toward scalable ion traps for quantum information processing". New J Phys 12, 033031 (2010).

[3] C. M. Shappert et al, "Spatially uniform single-qubit gate op- erations with near-field microwaves and composite pulse compensation" New J Phys 15, 083053 (2013).

[4] D. Kielpinski et al. "Integrated optics architecture for trapped-ion quantum information processing” Quant Inf Proc special issue. DOI 10.1007/s11128-015-1162-2 